\title{
A Real-World Glimpse into the Epidemiology of ALS in Canada
}

Can J Neurol Sci. 2013; 40: 625

Amyotrophic lateral sclerosis (ALS) is an adult-onset neurodegenerative disorder characterized by the death of motor neurons in the cortex, brainstem, and spinal cord. The result is progressive muscle weakness, atrophy, and death from respiratory paralysis, usually within three to five years of symptom onset. Diagnosis of ALS requires the presence of both upper and lower motor neuron features with disease progression, in the absence of evidence of another disease that may explain these signs. The El Escorial system outlines the criteria required for the diagnosis of suspected, possible, probable, and definite ALS based on clinical, electrophysiological, and neuropathological examination. At present, there is no cure for ALS and treatment is primarily with the drug Riluzole, which acts by blocking glutamate release.

The incidence rate of ALS is 1-3/100,000 people per year and it increases with age, with a peak incidence rate being observed in the 55-75 year old age group of 10.5 and 7.4/100,000 for men and women respectively ${ }^{1}$. Previous studies of ALS incidence and mortality have presented inconsistent results with regard to the trend of rates over the past several decades. Reported increases of ALS incidence and mortality rates in the past may have resulted from better case ascertainment, an aging population, or a true increase in the incidence and death rate ${ }^{2}$. Geographic differences in the distribution of ALS have been attributed to genetic and environmental factors and may also result from ascertainment bias due to differing access to health care. For example, the Midwest region of the United States has the highest rates of ALS associated death.

A number of environmental risk factors have been implicated in the etiology of ALS, yet none have been unequivocally identified as causal agents. The Neuromuscular Registry of the SLSJ would certainly be a great tool to study the various environmental factors that may influence rates of ALS such as heavy metals, pesticides, smoking, nutritional intake, physical activity, trauma or infectious agents.

Approximately $5-10 \%$ of ALS cases are familial (FALS), with the majority of cases deemed to be sporadic (SALS) in the absence of a positive family history. Given the numerous Mendelian forms of ALS, the involvement of genetic factors in the survival of motor neurons is indisputable. Many monogenic forms of ALS exist (SOD-1, FUS, TARDBP, C9orf72), often with wide genotypic and phenotypic heterogeneity. The C9orf72 hexanucleotide repeat expansion mutation has been observed in upwards of $9 \%$ of sporadic ALS cases in other population-based samples.

Patient registries are an important methodology for collecting real-world data for a variety of purposes including estimation of incidence and prevalence, clinical trial readiness and studies of natural history of both diseases and disease-specific outcome measures. The Irish Motor Neuron Disease Project previously demonstrates an ALS case ascertainment of $99 \%$ using sophisticated capture-recapture validation methodology ${ }^{3}$.
The study in the current issue by Lareau-Trudel et al describes epidemiologic surveillance of ALS in the SaguenayLac-St-Jean region of Quebec (SLSG) ${ }^{4}$. The authors report similar clinical features of ALS to those observed in the literature, but they also report a significant increase in incidence rate of ALS among the $>65$ years-old group between the 19852004 and the 2005-2009 periods. The reasons for this increase are not yet clear, since the study was performed in a small size population. It does not appear to be due to obvious biases such as aging of the population or greater access to neurologist care that would have improved ALS diagnosis over time.

The Quebec population of 7.5 million people contains about six million French-Canadians who are descendants of around 8000 French settlers who colonized "Nouvelle-France" between 1608 and 1765. There was sustained demographic growth, in a context of relative isolation, with doubling of the population every 25-30 years, thereby giving rise to a founder population undergoing rapid expansion. The SLSJ is populated by descendants of the initial French-Canadian founders, and this region is known for its high prevalence of certain genetic disorders as a result of founder effect. In addition to better studying the environmental factors causing ALS, the SLSJ region may represent a unique environment for conducting cutting-edge genomic research that may explain a significant proportion of cases in that population.

\author{
Nicolas Dupré ${ }^{1}$, Lawrence Korngut ${ }^{2}$ \\ ${ }^{1}$ Laval University, Quebec City, Quebec \\ ${ }^{2}$ University of Calgary, Calgary, Alberta
}

\section{REFERENCES}

1. Traynor BJ, Codd MB, Corr B, Forde C, Frost E, Hardiman O. Incidence and prevalence of ALS in Ireland, 1995-1997: a population-based study. Neurology. [Research Support, NonU.S. Gov't]. 1999 Feb;52(3):504-9.

2. Mehal JM, Holman RC, Schonberger LB, Sejvar JJ. Amyotrophic lateral sclerosis/motor neuron disease deaths in the United States, 1999-2009. Amyotroph Lateral Scler Frontotemporal Degener. 2013 Apr 29. [Epub ahead of print].

3. Donaghy C, Clarke J, Patterson C, Kee F, Hardiman O, Patterson V. The epidemiology of motor neuron disease in Northern Ireland using capture-recapture methodology. Amyotroph Lateral Scler. 2010 August; 11(4):374-8.

4. Lareau-Trudel E, Fortin E, Gauthier M, Lavoie S, Morissette E, Mathieu J. Epidemiological Surveillance of Amyotrophic Lateral Sclerosis in Saguenay Region. Can J Neurol Sci. 2013; 40(5):705-9. 